\title{
SPECTROSCOPIC PROPERTIES OF THE NUCLEUS OF NGC 6826
}

\author{
B. ALTNER \\ Applied Research Corporation, Landover, MD USA \\ S.R. HEAP \\ Laboratory for Astronomy and Solar Physics \\ NASA Goddard Space Flight Center, Greenbelt, MD USA \\ and \\ I. HUBENY \\ Universities Space Research Association (USRA) \\ NASA Goddard Space Flight Center, Greenbelt, MD USA
}

\begin{abstract}
We continue the trend of finding discrepancies in the values of central star masses derived from photospheric line profile fitting and other methods in our analyses of ground-based and UV spectra of the nucleus of NGC 6826.
\end{abstract}

Many apparent contradictions and uncertainties in the basic parameters of planetary nuclei cloud the general picture of the late stages of evolution of low- and intermediate-mass stars. The primary uncertainty is the mass distribution of the central stars. Different methods - non-LTE photospheric analyses (e.g., Mendez et al. 1988, Astr. Ap., 190, 113), analyses of the wind spectrum (e.g., Pauldrach et al. 1988 Astr. Ap., 207, 123), application of the Zanstra method, use of the optical fading diagram (e.g., Heap and Augensen 1987 Ap. J., 313, 268) - yield different results for the stellar mass, and there is no clear consensus as to which method, if any, is the correct one (Pottasch 1989, in "Planetary Nebulae", IAU Symp. No. 131, p. 481).

We have used high-dispersion ultraviolet and visual spectra to derive the properties of the O3Iaf*-type central star of the planetary nebula NGC 6826. Based on comparisons to models within an extensive grid of non-LTE atmospheres we conclude that the star has a temperature of $50,000 \mathrm{~K} \pm 5000$, and a $\log \mathrm{g} \approx 3.9$, yielding a mass $\approx 0.82 M_{\odot}$. However, both the strength of the He II $\lambda 4686$ emission line and the terminal velocity of the stellar wind (as determined from the P Cygni profiles of the strong UV resonance lines), suggest a mass near $0.65 M_{\odot}$. Application of our newest method of mass determination for central stars (optical-UV fading; see Altner and Heap, this volume) yields an upper limit of $0.64 \pm 0.1 M_{\odot}$ for NGC 6826. Even lower masses are found from the optical fading diagram and the Zanstra temperature and luminosity. Therefore, this star, like many Of-type central stars, shows a discrepancy between the mass derived from photospheric analysis and that derived from other methods. 